\title{
GENERALIZED ZETA-FUNCTIONS
}

\section{FOR AXIOM A BASIC SETS}

\author{
BY D. RUELLE
}

Communicated October 15,1975

Let $X$ be a set, $f: X \mapsto X$ a map, $\varphi: X \mapsto \mathrm{C}$ a complex-valued function. We write formally

$$
D(\varphi)=\exp \left[-\sum_{n=1}^{\infty} \frac{1}{n} \sum_{\xi \in \mathrm{Fix} f^{n}} \prod_{k=0}^{n-1} \varphi\left(f^{k} \xi\right)\right]
$$

Taking $\varphi$ constant, i.e. replacing $\varphi$ by $z \in \mathrm{C}$, we can interpret $1 / D(z)$ as a zetafunction proved to be rational for Axiom A diffeomorphisms by Guckenheimer and Manning [6].

Similarly, if $\left(f^{t}\right)$ is a flow on $X$, we write formally

$$
d(A)=\prod_{\gamma}\left[1-\exp \int_{0}^{\lambda(\gamma)} A\left(f^{t} x_{\gamma}\right) d t\right]
$$

where the product extends over the periodic orbits $\gamma$ of the flow, $\lambda(\gamma)$ is the prime period of $\gamma$ and $x_{\gamma}$ a point of $\gamma$.

In this note we indicate analyticity properties of $A \rightarrow D\left(e^{A}\right)$ or $A \rightarrow d(A)$ for diffeomorphisms or flows satisfying Smale's Axiom A, assuming only that $A$ is Hölder continuous. Our results hold in particular for Anosov diffeomorphisms and flows, and when $A$ is $C^{\mathbf{1}}$. Stronger properties of meromorphy hold under suitable assumptions of real-analyticity and will be published elsewhere by $\mathbf{P}$. Cartier and the author.

Let $\Lambda$ be a basic set for a $C^{\mathbf{1}}$ diffeomorphisms or flow satisfying Smale's Axiom A (see [13]). Choosing a Riemann metric $d$, and $\alpha \in(0,1)$ we let $C^{\alpha}$ be the Banach space of real Hölder continuous functions of exponent $\alpha$, with the norm

$$
\|A\|_{\alpha}=\sup \left\{|A(x)|+\frac{|A(y)-A(x)|}{(d(x, y))^{\alpha}}: x, y \in \Lambda \text { and } x \neq y\right\}
$$

We denote by $\mathcal{C}_{\mathbf{C}}^{\alpha}$ the corresponding space of complex functions.

1. TheOREM. Let the Axiom A diffeomorphism $f$ restricted to the basic set $\Lambda$ be topologically mixing. We denote by $P(A)$ the (topological) pressure of a real continuous function $A$ on $\Lambda$ (see [8], [14], [4] ). There is a continuous real function $R$ on $C_{\mathrm{C}}^{\alpha}$ satisfying

AMS (MOS) subject classifications (1970). Primary 58F20; Secondary 82A05. 


$$
\begin{aligned}
R(A) & \geqslant \exp [-P(\operatorname{Re} A)]>0, \\
R(A+c) & =e^{-\operatorname{Re} c} R(A) \quad \text { when } c \in \mathrm{C}
\end{aligned}
$$

and such that

(a) if $A \in \mathcal{C}_{\mathrm{C}}^{\alpha}$, the following power series in $z$,

$$
D\left(z e^{A}\right)=\exp \left[-\sum_{m=1}^{\infty} \frac{z^{m}}{m} \sum_{x \in \mathrm{Fix} f^{m}} \exp \sum_{k=0}^{m-1} A\left(f^{k} x\right)\right]
$$

converges for $|z|<R(A)$. The function $A \mapsto D\left(e^{A}\right)$ is analytic in $\left\{A \in \mathcal{C}_{\mathbf{C}}^{\alpha}\right.$ : $R(A)>1\}$.

(b) If $A \in \mathcal{C}^{\alpha}$, then $R(A)>\exp [-P(A)]$, and $z \mapsto D\left(z e^{A}\right)$ has only one zero in $\{z:|z|<R(A)\}$. This zero is simple and located at $\exp [-P(A)]$. on $f$.

We shall also write $P_{f}, R_{f}, D_{f}$ instead of $P, R, D$, to indicate the dependence

We outline the proof of Theorem 1. First suppose that $(\Lambda, f)$ is a subshift of finite type (see [13]). Then the theorem can be proved by the "transfer matrix" method of statistical mechanics (see [7], [1], [12], [9], [10]). The general case reduces to that one: using a Markov partition for $\Lambda$ (see [11], [2]) one can, by a combinatorial lemma of Manning [6], write

$$
D_{f}\left(z e^{A}\right)=\prod_{i \in I}\left[D_{\tau_{i}}\left(z e^{A \circ \pi_{i}}\right)\right]^{s_{i}} .
$$

In this formula the index set $I$ is finite, $s_{i}= \pm 1$, the $\tau_{i}$ are shifts acting on spaces $\Omega_{i}$ and the $\pi_{i}: \Omega_{i} \mapsto \Lambda$ are Holder continuous maps such that $\pi_{i} \tau_{i}=$ $f \pi_{i}$. Furthermore there is an index $1 \in I$ such that $s_{1}=+1$ and

$$
P_{f}=P_{\tau_{1}} \circ \pi_{1}>P_{\tau_{i}} \circ \pi_{i} \text { if } i \neq 1
$$

[ $\pi_{1}$ defines the symbolic dynamics associated with the Markov partition; therefore $P_{f}=P_{\tau_{1}} \circ \pi_{1}$ (see for instance [4]). If $i \neq 1, \pi_{i} \Omega_{i} \neq \Lambda$ and therefore the pressure of $f$ restricted to $\pi_{i} \Omega_{i}$ is $\left\langle P_{f}\right.$. This gives bounds on $f$-periodic points in $\pi_{i} \Omega_{i}$, and therefore on $\tau_{i}$-periodic points in $\Omega_{i}$, implying $P_{\tau_{i}} \circ \pi_{1}>P_{\tau_{i}} \circ$ $\left.\pi_{i}\right]$. The conditions of the theorem are satisfied if we take

$$
R_{f}(A)=\min \left\{R_{\tau_{1}}\left(A \circ \pi_{1}\right), \min _{i \neq 1} \exp \left(-P_{\tau_{i}}\left(\operatorname{Re} A \circ \pi_{i}\right)\right)\right\} .
$$

CoRollary. $P$ is a real-analytic function on $C^{\alpha} ; e^{P(A)}$ is the radius of convergence of the series

$$
\sum_{m=1}^{\infty} \frac{z^{m}}{m} \sum_{x \in K_{m}} \exp \sum_{k=0}^{m-1} A\left(f^{k} x\right)
$$


where $K_{m}$ consists of the f-periodic points of prime period $m$.

2. Theorem. Let $\Lambda$ be a basic set for an Axiom A flow $\left(f^{t}\right)$. We denote by $P(A)$ the topological pressure of a real continuous function $A$ on $\Lambda$ (see [5]). There is a continuous real function $r \geqslant 0$ on $C_{\mathrm{C}}^{\alpha}$ such that:

(a) if $A \in \mathcal{C}_{\mathrm{C}}^{\alpha}$, the product

$$
d(A-u)=\prod_{\gamma}\left[1-\exp \int_{0}^{\lambda(\gamma)}\left(A\left(f^{t} x_{\gamma}\right)-u\right) d t\right]
$$

is convergent for $\operatorname{Re} u>P(\operatorname{Re} A)$ and extends to an analytic function of $u$ for $|u-P(\operatorname{Re} A)|<r(A)$. The function $d$ is analytic in $\left\{A \in \mathcal{C}^{\alpha}: P(\operatorname{Re} A)<r(A)\right\}$;

(b) If $A \in C^{\alpha}$, then $r(A)>0$, and $u \mapsto d(A-u)$ has only one zero in $\{u: \operatorname{Re} u>P(A)$ or $|u-P(A)|<r(A)\}$. This zero is simple and located at $P(A)$.

The proof is based on a technique of counting periodic orbits due to Bowen [3, §5].

Corollary. $P$ is a real-analytic function on $C^{\alpha} ; P(A)$ is the abscissa of convergence of the Dirichlet series $\Sigma_{\gamma} \exp \int_{0}^{\lambda(\gamma)}\left(A\left(f^{t} x_{\gamma}\right)-u\right) d t$.

REMARK. The functions $z \longmapsto D\left(z e^{A}\right)$ of Theorem 1 and $u \longmapsto d(A-u)$ of Theorem 2 do not in general extend to meromorphic functions in the whole complex plane. Counterexamples have been constructed by G. Gallavotti (private communication).

\section{REFERENCES}

1. H. Araki, Gibbs states of a one dimensional quantum lattice, Comm. Math. Phys. 14 (1969), 120-157. MR 42 \#8834.

2. R. Bowen, Markov partitions for Axiom A diffeomorphisms, Amer. J. Math. 92 (1970), 725-747. MR 43 \#2740.

3. Symbolic dynamics for hyperbolic flows, Amer. J. Math. 95 (1973), 429460. MR 49 \#4041.

(to appear).

4. - Equilibrium states and the ergodic theory of Anosov diffeomorphisms

5. R. Bowen and D. Ruelle, The ergodic theory of Axiom A flows, Invent. Math., 29 (1975), $181-202$.

6. A. Manning, Axiom A diffeomorphisms have rational zeta functions, Bull. London Math. Soc. 3 (1971), 215-220. MR 44 \#5982.

7. D. Ruelle, Statistical mechanics of a one-dimensional lattice gas, Comm. Math. Phys. 9 (1968), 267-278. MR 38 \#3013.

8. - Statistical mechanics on a compact set with $\mathrm{Z}^{\nu}$-action satisfying expansiveness and specification, Bull Amer. Math. Soc. 78 (1972), 988-991; Trans. Amer. Math. Soc. 185 (1973), 237-251. MR 47 \#292.

9. - A measure associated with Axiom A attractors, Amer. J. Math. (to appear).

10. - Notes on classical statistical mechanics (to appear).

11. Ja. G. Sină, Construction of Markov partitionings, Funkcional. Anal. i Priložen. 2 (1968), no. 3, 70-80 = Functional Anal. Appl. 2 (1968), 39-52. MR 40 \#351. 
12. Ja. G. Sinaĩ, Gibbsian measures in ergodic theory, Uspehi Mat. Nauk 27 (1972), no. 4 (166), 21-64 = Russian Math. Surveys 27 (1972), no. 4, 21-70.

13. S. Smale, Differentiable dynamical systems, Bull. Amer. Math. Soc. 73 (1967), 747-817. MR 37 \#398; erratum 139, p. 1593.

14. P. Walters, $A$ variational principle for the pressure of continuous transformations (to appear). FRANCE

INSTITUT DES HAUTES ETUDES SCIENTIFIQUES, BURES-SUR-YVETTE,

BULLETIN OF THE

AMERICAN MATHEMATICAL SOCIETY

Volume 82, Number 1, January 1976

\section{ERRATUM, VOLUME 81}

On p. 823 of the September 1975 Bulletin the name of Robert L. Anderson was inadvertently included as a panel member for the AMS-MAA Committee on the Training of Graduate Students to Teach. He should have been listed as a panel member for the AMS Committee on Employment and Educational Policy discussion on "Seeking employment outside academia: Views from some who have recently succeeded". 